\title{
Chebyshev Polynomials Corresponding to a Semi-Infinite Interval and an Exponential Weight Factor
}

\author{
By David W. Kammler
}

\begin{abstract}
An algorithm is presented for the computation of the $n$ zeros of the polynomial $q_{n}$ having the property that $q_{n}(t) \exp (-t)$ alternates $n$ times, at the maximum value 1 , on $[0,+\infty)$. Numerical values of the zeros and extremal points are given for $n \leqq 10$.
\end{abstract}

1. Introduction. Using well-known arguments from the theory of minimax approximation (cf. [2, pp. 28-31]), it can be shown that for each $n=0,1,2, \cdots$ there exists a unique polynomial $q_{n}$ of degree $n$ and $n+1$ real numbers $0=\tau_{n 0}<\tau_{n 1}<\cdots$ $<\tau_{n n}$ such that

$$
\begin{gathered}
\max \left\{\left|q_{n}(t) \exp (-t)\right|: t \geqq 0\right\}=1, \\
q_{n}\left(\tau_{n k}\right) \exp \left(-\tau_{n k}\right)=(-1)^{n-k}, \quad k=0,1, \cdots, n,
\end{gathered}
$$

i.e., such that $q_{n}$ is the Chebyshev polynomial of degree $n$ which corresponds to the semi-infinite interval $[0,+\infty)$ and to the weight function $w(t)=\exp (-t)$.

By means of a zero counting argument, we see that whenever $y$ satisfies the differential equation

$$
(D+1)^{n+1} y(t)=0, \quad t \geqq 0, D=d / d t
$$

and the normalization condition

$$
\max \{|y(t)|: t \geqq 0\} \leqq 1,
$$

then

$$
|y(t)| \leqq q_{n}(t) \exp (-t) \quad \text { for } t \geqq \tau_{n n},
$$

with equality possible only if

$$
y(t)= \pm q_{n}(t) \exp (-t), \quad t \geqq 0 .
$$

Moreover, it can be shown [1, Theorem 2] that (5) also holds whenever $y$ is any solution of the more general differential equation

(7) $\left[\left(D-\lambda_{0}\right) \cdots\left(D-\lambda_{n}\right)\right] y(t) \equiv 0$ for $t \geqq 0$ with $-\infty<\lambda_{0}, \cdots, \lambda_{n} \leqq-1$

Received August 8, 1972.

AMS (MOS) subject classifications (1970). Primary 41A50, 65D20.

Key words and phrases. Zeros and extremal points of Chebyshev polynomial on $[0,+\infty)$ with exponential weight, minimal decay rate for exponential sum. 
which is subject to (4), again with equality possible only if $y$ is given by (6). In particular, if $y$ satisfies (7), then

$$
|y(t)| \leqq \max \left\{|y(s)|: 0 \leqq s \leqq \tau_{n n}\right\} q_{n}(t) \exp (-t) \quad \text { for } t \geqq \tau_{n n} .
$$

Thus, the familiar "maximum growth" property [3, Theorem 6, p. 51] of the ordinary Chebyshev polynomials (associated with the interval $[-1,1]$ and the weight function $w(t) \equiv 1)$ corresponds to the "minimal decay rate" (8) for any transient satisfying (7).

By using (8) and the particular function $y(t)=t^{n} \exp (-t), t \geqq 0$, which takes its maximum at $t=n$, we conclude that $\tau_{n n} \geqq n, \quad n=1,2, \cdots$. No simple upper bound for $\tau_{n n}$ (which could replace $\tau_{n n}$ in (8)) is presently known, although we conjecture that $\tau_{n n} \leqq 2 n$ for all $n$ as is certainly the case for $n \leqq 40$ (as we have verified numerically).

2. Numerical Determination of $q_{n}$. Let $n \geqq 1$ be fixed and let $\mathrm{z}=\left(z_{1}, \cdots, z_{n}\right)$ with $0<z_{1}<\cdots<z_{n}$ be given estimates of the zeros of $q_{n}$. We define

$$
\varphi(\mathrm{z}, t)=\left[\left(t / z_{1}-1\right) \cdots\left(t / z_{n}-1\right)\right] \exp (-t), \quad t \geqq 0,
$$

and seek to adjust the parameters $\mathrm{z}$ so as to level $\varphi$ and thereby force $\varphi$ to satisfy the normalization condition (4).

For $i=1, \cdots, n$, we let $t_{i}(\mathrm{z})$ denote the unique point where $|\varphi(\mathrm{z},-)|$ takes its maximum on the interval $\left(z_{i}, z_{i+1}\right)$ (with $z_{n+1}$ defined to be $+\infty$ ). Given $\mathbf{z}$, we may numerically determine $t_{i}(\mathbf{z})$ by using standard rootfinding techniques (e.g., bisection followed by Newton's method) to locate the unique zero of the function

$$
\varphi_{t}(\mathrm{z}, t) / \varphi(\mathrm{z}, t)=\left(t-z_{1}\right)^{-1}+\cdots+\left(t-z_{n}\right)^{-1}-1, \quad z_{i}<t<z_{i+1}
$$

(with the subscript denoting the corresponding partial derivative).

The perturbation $\mathrm{h}(\mathrm{z})=\left(h_{1}(\mathrm{z}), \cdots, h_{n}(\mathrm{z})\right)$ is defined in such a manner that

$$
\varphi\left(\mathrm{z}+\mathrm{h}(\mathrm{z}), t_{i}(\mathrm{z})\right) \approx(-1)^{n-i}, \quad i=1, \cdots, n,
$$

to terms of first order in $h(z)$, i.e., such that

$$
\varphi\left(\mathrm{z}, t_{i}(\mathrm{z})\right)+\sum_{i=1}^{n} \varphi_{z_{i}}\left(\mathrm{z}, t_{i}(\mathrm{z})\right) h_{j}(\mathrm{z})=(-1)^{n-i}, \quad i=1, \cdots, n .
$$

Using (9) in (11), we obtain the equivalent system of linear equations

$$
\sum_{j=1}^{n} \frac{h_{j}(\mathrm{z})}{z_{i}\left[t_{i}(\mathrm{z})-z_{i}\right]}=\frac{\varphi\left(\mathrm{z}, t_{i}(\mathrm{z})\right)-(-1)^{n-i}}{t_{i}(\mathrm{z}) \varphi\left(\mathrm{z}, t_{i}(\mathrm{z})\right)}, \quad i=1, \cdots, n,
$$

which may be used to compute $\mathbf{h}(\mathbf{z})$ when $\mathbf{z}$ is given. (Indeed, since any linear combination of the $n$ functions $\psi_{i}(t)=\left(t-z_{i}\right)^{-1}, t \neq z_{i}, i=1, \cdots, n$, can be expressed as the ratio of two polynomials with the numerator having degree at most $n-1$, it follows that no such linear combination can have more than $n-1$ zeros. Thus, the columns of the coefficient matrix in (12) are linearly independent so that (12) uniquely determines $\mathbf{h}(\mathbf{z})$.)

This being the case, we may begin with a suitable initial estimate, $z_{1}$, and then successively compute 


$$
\mathrm{z}_{v+1}=\mathrm{z}_{\nu}+\mathrm{h}\left(\mathrm{z}_{\nu}\right), \quad \nu=1,2, \cdots,
$$

in hopes that this sequence will converge to the limit $\zeta=\left(\zeta_{n 1}, \cdots, \zeta_{n n}\right)$ where $\zeta_{n 1}<\cdots$ $<\zeta_{n n}$ are the (positive) zeros of $q_{n}$. The sequence will certainly converge to $\zeta$ provided that $z_{1}$ is sufficiently close to $\zeta$. Indeed, using (9), (10), (12) and the implicit function theorem we see that $t_{i}(\mathrm{z})$ and $h_{i}(\mathrm{z}), i=1, \cdots, n$, are all continuously differentiable functions of $\mathbf{z}$ in some neighborhood of $z=\zeta$ so that we may write

$$
h_{j}(\mathrm{z})=h_{i}(\zeta)+\sum_{k=1}^{n} \frac{\partial h_{j}}{\partial z_{k}}(\zeta)\left(z_{k}-\zeta_{n k}\right)+o(|z-\zeta|), \quad j=1, \cdots, n .
$$

Since $h(z)$ corresponds to a perturbation about $z=\zeta$ and since $t_{i}(z)$ is an extreme point of $\varphi$, we have

$$
\begin{aligned}
h_{i}(\zeta) & =0, \quad j=1, \cdots, n, \\
\varphi_{l}\left(\zeta, t_{i}(\zeta)\right) & =0, \quad i=1, \cdots, n,
\end{aligned}
$$

and, by making use of these identities in the equation which results when (11) is differentiated with respect to $z_{k}$, we obtain

$$
\partial h_{i}(\zeta) / \partial z_{k}=-\delta_{i k}, \quad j, k=1, \cdots, n .
$$

Thus, (14) reduces to

$$
\mathbf{h}(\mathbf{z})=\zeta-\mathbf{z}+o(|z-\zeta|) .
$$

Using (13), (15) and considerations of continuity, we conclude that $\left\{\boldsymbol{z}_{v}\right\}$ converges to $\zeta$ and that $\left\{t_{k}\left(\mathbf{z}_{v}\right)\right\}$ converges to the $k$ th extremal point $\tau_{n k}$ of (2) for $q_{n}$ provided that $z_{1}$ is sufficiently close to $\zeta$. (A slight extension of the above argument shows that the convergence is quadratic in each case.)

3. Numerical Results. Using the above procedure, we have computed the zeros $\zeta_{n k}$ and the extremal points $\tau_{n k}$ for $n \leqq 40$, and we list our (rounded) results for $n \leqq 10$ in Table 1 . The roots $\zeta_{n k}, k=1, \cdots, n$, can be modeled relatively well by

$$
\begin{aligned}
& z_{1}=.308 / n-.026 / n^{2}, \\
& z_{2}=z_{1} / .111, \\
& z_{k}=z_{k-1} /\left\{1-2.04 / k+.34 / k^{2}-.10 /(n+2-k)\right\}, \quad k=3, \cdots, n,
\end{aligned}
$$

and for $n \leqq 40$ about a half dozen iterations are needed to locate the zeros and extreme points of $q_{n}$ to 16 place accuracy when these initial estimates are used. Finally, we note that the leading coefficient, $a_{n}$, of $q_{n}$ (which corresponds to the leading coefficient $2^{1-n}$ for the ordinary $n$th order Chebyshev polynomial) appears to decay with $n$ in such a manner that

$$
\begin{array}{r}
a_{n}=\left(\zeta_{n 1} \cdots \zeta_{n n}\right)^{-1} \approx\{\alpha[\alpha+1 / 2] \cdots[\alpha+(n-1) / 2]\}^{-1}, \\
\alpha=.276, n=1,2, \cdots,
\end{array}
$$

with this approximation being good to within about two percent for $n \leqq 40$. 
TABLE 1

Zeros and Extremal Points for $q_{n}$

\begin{tabular}{|c|c|c|c|c|c|}
\hline$n$ & $\zeta_{n k}$ & $\tau_{n k}$ & $n$ & $\zeta_{n k}$ & $\tau_{n k}$ \\
\hline \multirow[t]{3}{*}{1} & .27846 & 0.00000 & 7 & 0.04364 & 0.00000 \\
\hline & & 1. 27846 & & 0.39600 & 0.17509 \\
\hline & & & & 1.11914 & 0.70924 \\
\hline \multirow[t]{4}{*}{2} & 0.14728 & 0.00000 & & 2.25574 & 1.63180 \\
\hline & 1.47277 & 0.61035 & & 3.88966 & 3.00276 \\
\hline & & 3.00971 & & 6.19599 & 4.94116 \\
\hline & & & & 9.65118 & 7.72085 \\
\hline \multirow[t]{5}{*}{3} & 0.09996 & 0.00000 & & & 12.37043 \\
\hline & 0.94116 & 0.40635 & & & \\
\hline & 2.94440 & 1.75198 & 8 & 0.03824 & 0.00000 \\
\hline & & 4.82719 & & 0.34636 & 0.15333 \\
\hline & & & & 0.97479 & 0.61924 \\
\hline \multirow[t]{6}{*}{4} & 0.07561 & 0.00000 & & 1.95077 & 1.41689 \\
\hline & 0.69785 & 0.30523 & & 3. 32411 & 2.58340 \\
\hline & 2.05438 & 1. 27074 & & 5.18551 & 4. 18557 \\
\hline & 4.53706 & 3. 10443 & & 7.71882 & 6. 34983 \\
\hline & & 6.68449 & & 11.41884 & 9.36171 \\
\hline & & & & & 14.28748 \\
\hline \multirow[t]{7}{*}{5} & 0.06078 & 0.00000 & & & \\
\hline & 0.55591 & 0.24456 & 9 & 0.03403 & 0.00000 \\
\hline & 1. 59954 & 1.00310 & & 0.30782 & 0.13638 \\
\hline & 3.33784 & 2.36634 & & 0.86390 & 0.54968 \\
\hline & 6.19974 & 4.57439 & & 1.72079 & 1. 25311 \\
\hline & & 8.56540 & & 2.91087 & 2. 27144 \\
\hline & & & & 4. 48859 & 3.64662 \\
\hline \multirow[t]{16}{*}{6} & 0.05080 & 0.00000 & & 6.54969 & 5.45012 \\
\hline & 0.46240 & 0.20406 & & 9.28522 & 7.81416 \\
\hline & 1. 31541 & 0.83046 & & 13. 20644 & 11.03436 \\
\hline & 2.68315 & 1.92781 & & & 16.21148 \\
\hline & 4. 72922 & 3.60468 & & & \\
\hline & 7.90880 & 6.12060 & 10 & 0.03066 & 0.00000 \\
\hline & & 10.46217 & & 0.27702 & 0.12281 \\
\hline & & & & 0.77592 & 0.49426 \\
\hline & & & & 1.54056 & 1. 12387 \\
\hline & & & & 2.59332 & 2.02907 \\
\hline & & & & 3.96994 & 3.23820 \\
\hline & & & & 5.72826 & 4.79650 \\
\hline & & & & 7.96803 & 6.77906 \\
\hline & & & & 10.88659 & 9.32290 \\
\hline & & & & 15.01021 & 12.73268 \\
\hline & & & & & 18.14115 \\
\hline
\end{tabular}


Department of Mathematics Southern Illinois University

Carbondale, Illinois 62901

1. D. W. KAMmLer, "A minimal decay rate for solutions of stable $n$-th order homogeneous differential equations with constant coefficients." (To appear.)

2. G. Meinardus, Approximation of Functions: Theory and Numerical Methods, Springer, Berlin, 1964; English transl., Springer Tracts in Natural Philosophy, vol. 13, SpringerVerlag, New York, 1967. MR 31 \# 547; 36 \#571.

3. I. P. Natanson, Constructive Function Theory. Vol. I. Uniform Approximation, Ungar, New York, 1964. MR 33 \#4529a. 